\title{
Comportamento reológico das polpas de noni integral e concentradas
}

\author{
Rheological behavior of whole and concentrated noni pulp
}

\section{Sonara de França Sousa1*, Alexandre José de Melo Queiroz¹, Rossana Maria Feitosa de Figueirêdo', Francilânia Batista da Silva ${ }^{1}$}

${ }^{1}$ Universidade Federal de Campina Grande (UFCG), Unidade Acadêmica de Engenharia Agrícola, Campina Grande/PB - Brasil

\section{${ }^{*}$ Corresponding Author}

Sonara de França Sousa, Universidade Federal de Campina Grande (UFCG), Unidade Acadêmica de Engenharia Agrícola, Avenida Aprígio Veloso, 882, CEP: 58429-970, Campina Grande/PB - Brasil, e-mail: sonara_franca@yahoo.com.br

Cite as: Rheological behavior of whole and concentrated noni pulp. Braz. J. Food Technol., v. 20, e2016067, 2017.

Received: June 17, 2016; Accepted: Mar. 03, 2017

\section{Resumo}

O comportamento reológico de polpas de frutas permite a obtenção de importantes informações sobre seu escoamento em distintas condições e nas diversas etapas do processo. Em vista disso, o presente trabalho teve como objetivo analisar o comportamento reológico da polpa de noni (Morinda citrifolia L.) integral e concentradas a 30 e 50Brix. Os parâmetros reológicos determinados foram: tensão de cisalhamento, taxa de deformação e viscosidade aparente, utilizando-se um viscosímetro Brookfield nas velocidades de rotação de 5 a $200 \mathrm{rpm}$ e temperaturas de 5 a $65^{\circ} \mathrm{C}$. Dentre os modelos testados, 0 que melhor representou os reogramas das polpas de noni integral e concentradas foi o de Mizrahi \& Berk, cujos ajustes foram $R^{2} \geq 0,8219, X^{2} \leq 0,1569$ e $P \leq 2,26 \%$. As polpas estudadas apresentaram valores para índice de comportamento do fluido menores que um $(n<1)$, sendo caracterizadas, portanto, como fluidos não newtonianos com comportamento pseudoplástico. A equação do tipo Arrhenius pode ser utilizada para demonstrar o efeito da temperatura sobre a viscosidade aparente nas polpas integral e concentrada com $30^{\circ}$ Brix, em todas as velocidades de rotação, e na polpa concentrada com $50^{\circ}$ Brix, preferencialmente nas velocidades de rotação de 5, 10 e 20 rpm.

Palavras-chave: Morinda citrifolia L.; Fluido pseudoplástico; Viscosidade aparente.

\section{Summary}

The rheological behaviour of fruit pulps allows one to obtain important information about their flow under distinct conditions and at different stages of the process. Thus the present study aimed to analyse the rheological behaviour of whole noni pulp (Morinda citrifolia L.) and that concentrated to 30 and $50{ }^{\circ}$ Brix. The rheological parameters determined were: shear stress, strain rate and apparent viscosity, using a Brookfield viscometer at rotational speeds of 5 to $200 \mathrm{rpm}$ and temperatures from 5 to $65^{\circ} \mathrm{C}$. Of the models tested, the Mizrahi \& Berk model best represented the rheograms of the whole and concentrated noni pulps with the following fittings: $R^{2} \geq 0.8219, X^{2} \leq 0.1569$ and $P \leq 2.26 \%$. The pulps studied presented values for the fluid behaviour index of less than one $(n<1)$, characterizing them as non-Newtonian fluids with pseudoplastic behaviour. The Arrhenius equation can be used to demonstrate the effect of temperature on the apparent viscosity for the whole and $30^{\circ} \mathrm{Brix}$ pulps at all speeds, and for the pulp with $50^{\circ}$ Brix preferably at rotational speeds of 5, 10 and $20 \mathrm{rpm}$.

Keywords: Morinda citrifolia L.; Pseudoplastic fluid; Apparent viscosity.

\section{Introdução}

A Morinda citrifolia L. é um planta pertencente à família Rubiaceae, conhecida popularmente como noni. É originária do sudoeste da Ásia e seu cultivo e consumo têm se expandido rapidamente em todas as regiões brasileiras, não apenas por ser uma rica fonte de nutrientes, mas principalmente devido às suas propriedades fitoterápicas e à sua facilidade de adaptação (NASCIMENTO, 2012). Os frutos apresentam formatos diformes a ovalados, com coloração da casca branca, quando maduros, polpa mole e friável, da qual é desprendido um forte odor que, segundo Pino et al. (2009), é possivelmente causado pela presença de ácidos orgânicos, entre os quais os ácidos octanoico e etanoico. 
As questões relacionadas ao manuseio de frutas em escala industrial têm, como uma das soluções mais práticas, a transformação da matéria-prima em polpa, viabilizando a utilização em inúmeros processos (OLIVEIRA et al., 2012). Neste contexto, torna-se imprescindível o estudo reológico, o qual correlaciona a tensão e a deformação, sendo capaz de descrever as propriedades do material em questão baseando-se em parâmetros derivados destas relações (ZHONG; DAUBERT, 2007). A viscosidade é o principal parâmetro estudado em alimentos líquidos e semilíquidos, e é considerada o meio de fundamento para se caracterizar a textura do fluido. Na industrialização de polpa de frutas, frequentemente são utilizados processos térmicos (aquecimento e/ou resfriamento), que podem acarretar modificação na sua viscosidade, o que faz com que o estudo da influência da temperatura sobre o comportamento reológico desse produto seja de grande importância (FEITOSA et al., 2015).

A descrição do comportamento reológico é feita através de modelos empíricos e semiempíricos, que são usados para relacionar a tensão de cisalhamento com a taxa de deformação, facilitando os cálculos de engenharia. $\mathrm{Na}$ literatura, há diversas equações que descrevem o comportamento não newtoniano de fluidos e, entre as mais utilizadas, encontram-se os modelos de Ostwald-de-Waelle, Herschell-Buckley e Mizrahi \& Berk. Possibilita-se, a partir desses conhecimentos, adequar o controle das linhas de produção, o projeto e o dimensionamento dos processos (BRANCO, 1995).

Pesquisas relacionadas às propriedades reológicas da polpa do noni produzida no Brasil são escassas. Visando suprir esta carência de dados, o objetivo do presente trabalho foi determinar o comportamento reológico de polpas de noni integral e concentradas (30 e $50^{\circ}$ Brix) submetidas a distintas temperaturas e taxas de deformações, além de ajustar os dados aos modelos reológicos estudados e avaliar a influência da temperatura sobre a viscosidade aparente das amostras.

\section{Material e métodos}

Foram utilizados, como matéria-prima, nonis (Morinda citrifolia L.) em estágio de maturação maduros. Os frutos foram lavados em água corrente, sanitizados em água clorada (100 ppm) por 15 minutos, descascados manualmente com o auxílio de facas de aço inoxidável e a polpa integral foi separada das sementes através da prensagem em prensa hidráulica. Em seguida, a polpa integral foi acondicionada em sacos de polietileno de baixa densidade e armazenada em freezer a uma temperatura de aproximadamente $-20^{\circ} \mathrm{C}$. Uma parte da polpa integral foi concentrada em evaporador rotativo (QUIMIS, modelo Q-344B2) a $70-75^{\circ} \mathrm{C}$, até atingir as concentrações de sólidos solúveis totais (SST) de 30 e 50Brix, medidas através da leitura direta em refratômetro portátil do tipo ABBÉ.

\subsection{Comportamento reológico}

Os parâmetros reológicos obtidos foram: tensão de cisalhamento, taxa de deformação e viscosidade aparente, em triplicata, para a polpa integral e polpas concentradas de noni, utilizando-se um viscosímetro Brookfield digital DV-II+PRO, modelo RVT, nas velocidades de rotação de $5,10,20,40,60,80,100,120,140,150,160,180$ e 200 rpm, e temperaturas de $5,15,25,35,45,55$ e $65^{\circ} \mathrm{C}$, medidas com o auxílio de um termostato, à pressão atmosférica ambiente. Para transformar as leituras dos torques em medidas reológicas, utilizou-se a metodologia proposta por Mitschka (1982).

Com os dados de tensão de cisalhamento em função da taxa de deformação, foram traçados os reogramas e ajustados os modelos reológicos de Ostwald-de-Waelle ou Lei de Potência (Equação 1), Herschel-Bulkley (Equação 2) e Mizrahi \& Berk (Equação 3), utilizando-se o programa Statistica versão 5.0:

$$
\begin{aligned}
& \tau=\mathrm{K} \dot{\gamma}^{\mathrm{n}} \\
& \tau-\tau_{0 \mathrm{H}}=\mathrm{K}_{\mathrm{H}} \dot{\gamma}^{\mathrm{n}_{\mathrm{H}}} \\
& \tau^{0,5}=\mathrm{K}_{\mathrm{OM}}+\mathrm{K}_{\mathrm{M}} \dot{\gamma}^{n_{\mathrm{M}}}
\end{aligned}
$$

Em que:

$\tau$ - tensão de cisalhamento $(\mathrm{Pa})$;

$\tau_{O H}$ - tensão de cisalhamento inicial do modelo de HerschelBulkley $(\mathrm{Pa})$;

$\mathrm{K}_{\mathrm{om}}$ - raiz quadrada da tensão inicial $(\mathrm{Pa})$;

$\dot{\gamma}$ - taxa de deformação $\left(\mathrm{s}^{-1}\right)$;

$\mathrm{K}, \mathrm{K}_{\mathrm{H}}, \mathrm{K}_{\mathrm{M}}$ - índices de consistência $\left(\mathrm{Pa} . \mathrm{S}^{\mathrm{n}}\right)$;

$\mathrm{n}$ - índice de comportamento do fluido (adimensional).

Para avaliar o ajuste dos modelos reológicos aos dados coletados, foram calculados os valores dos coeficientes de determinação $\left(R^{2}\right)$, que é a porcentagem de variação total explicada, foi aplicado o teste de Qui-quadrado (Equação 4) e determinados os desvios percentuais médios (P\%) (Equação 5). Assim, o modelo reológico mais adequado para predizer o comportamento das polpas, é aquele com valores de $R^{2}$ próximos a um e baixos valores de $\mathrm{X}^{2}$ e $\mathrm{P} \%$.

$$
X^{2}=\sum(\text { Yobs }- \text { Ypre })^{2}
$$

Em que:

$X^{2}$ = teste do Qui-quadrado;

$Y$ obs = valor experimental;

$\mathrm{Y}$ pre $=$ valor previsto pelo modelo . 
$P=\frac{100}{n} \sum_{n=1}^{n}\left|\frac{\text { XeExp }- \text { XeTeor }}{\text { XeExp }}\right|$

Em que:

$P$ - desvio percentual médio (\%);

XeExp - valores obtidos experimentalmente;

XeTeor - valores preditos pelo modelo;

n - número de dados experimentais.

Foram traçadas as curvas da viscosidade aparente das polpas em função das velocidades de rotação, para todas as temperaturas analisadas. Para avaliar o efeito da temperatura sobre a viscosidade aparente, utilizou-se uma equação do tipo Arrhenius (Equação 6). A energia de ativação foi calculada a partir da regressão linear do gráfico do logaritmo neperiano da viscosidade aparente em função do inverso da temperatura absoluta $\left(\ln \eta_{a} \times 1 / T\right)$, utilizando-se o programa Origin Pro versão 8.0.

$\eta_{\mathrm{a}}=\eta_{0} \exp \left(\frac{\mathrm{Ea}}{\mathrm{RT}}\right)$

\section{Em que:}

$\eta_{a}$ - viscosidade aparente (Pa.s);

$\eta_{0}$ - viscosidade aparente inicial teórica (Pa.s);
$\mathrm{E}_{\mathrm{a}}$ - energia de ativação $\left(\mathrm{kJ} \mathrm{mol}^{-1}\right)$;

$\mathrm{R}$ - constante universal dos gases $\left(0,008314 \mathrm{~kJ} \mathrm{~mol}^{-1} \mathrm{~K}^{-1}\right)$;

$\mathrm{T}$ - temperatura absoluta (K).

\section{Resultados e discussão}

Nas Tabelas 1-3, são mostrados os parâmetros, os coeficientes de determinação $\left(R^{2}\right)$, os valores de Qui-quadrado $\left(\mathrm{X}^{2}\right)$ e os desvios percentuais médios ( $\mathrm{P} \%$ ) dos modelos reológicos de Ostwald-de-Waelle, Herschel-Bulkley e Mizrahi \& Berk, ajustados aos reogramas das polpas integral e concentradas de noni. Observa-se que todos os modelos testados apresentaram bons ajustes, com $\mathrm{R}^{2}$ $\geq 0,8219, X^{2} \leq 6,7021$ e $P \leq 10,18 \%$.

De acordo com os valores de $\mathrm{R}^{2}, \mathrm{X}^{2}$ e P\%, o melhor modelo para representar os reogramas das polpas de noni integral e concentradas (30 e $50^{\circ}$ Brix) foi o de Mizrahi \& Berk, apresentando $R^{2} \geq 0,8219, X^{2} \leq 0,1569$ e $P \leq 2,26 \%$. Os melhores ajustes também foram verificados com 0 modelo de Mizrahi \& Berk por Braga et al. (2013), ao avaliarem o comportamento reológico do suco de abacaxi cv. Pérola natural e tratado enzimaticamente, cujos valores de $\mathrm{R}^{2}$ foram maiores que 0,98 para todas as amostras analisadas, além de baixos valores de $\chi^{2}$; Ongaratto e

Tabela 1. Parâmetros, coeficientes de determinação $\left(R^{2}\right)$, valores de Qui-quadrado $\left(X^{2}\right)$ e desvios percentuais médios (P\%) dos modelos reológicos ajustados aos reogramas da polpa integral de noni.

\begin{tabular}{|c|c|c|c|c|c|c|c|}
\hline \multirow{2}{*}{ Modelo } & \multirow{2}{*}{ Temp. $\left({ }^{\circ} \mathbf{C}\right)$} & \multicolumn{2}{|c|}{ Parâmetros } & \multirow{2}{*}{\multicolumn{2}{|c|}{$\mathbf{R}^{2}$}} & \multirow{2}{*}{$\mathbf{X}^{2}$} & \multirow{2}{*}{$\mathbf{P}(\%)$} \\
\hline & & $K(\text { Pa.s })^{n}$ & $n$ & & & & \\
\hline \multirow{7}{*}{$\begin{array}{l}\text { Ostwald-de-Waelle } \\
\text { (Lei da Potência) }\end{array}$} & 5 & 1,7462 & 0,4126 & & 0,9823 & 0,1066 & 5,23 \\
\hline & 15 & 1,5281 & 0,4098 & & 0,9878 & 0,0563 & 4,58 \\
\hline & 25 & 1,1728 & 0,4191 & & 0,9785 & 0,0691 & 5,08 \\
\hline & 35 & 1,0128 & 0,4156 & & 0,9632 & 0,0849 & 7,13 \\
\hline & 45 & 0,7465 & 0,4813 & & 0,9721 & 0,0696 & 7,48 \\
\hline & 55 & 0,5659 & 0,5118 & & 0,9767 & 0,0469 & 7,80 \\
\hline & 65 & 0,5780 & 0,4824 & & 0,9712 & 0,0448 & 7,94 \\
\hline \multirow{8}{*}{ Herschel-Bulkley } & Temp. $\left({ }^{\circ} \mathrm{C}\right)$ & $\tau_{\mathrm{OH}}(\mathbf{P a})$ & $K_{H}(\text { Pa.s })^{n}$ & $\mathbf{n}_{\mathrm{H}}$ & $\mathbf{R}^{2}$ & $\mathbf{X}^{2}$ & P (\%) \\
\hline & 5 & 2,3386 & 0,3819 & 0,7212 & 0,9988 & 0,0071 & 0,97 \\
\hline & 15 & 1,8144 & 0,4401 & 0,6567 & 0,9992 & 0,0039 & 1,03 \\
\hline & 25 & 1,6227 & 0,2639 & 0,7158 & 0,9932 & 0,0219 & 2,49 \\
\hline & 35 & 1,7642 & 0,1034 & 0,8820 & 0,9953 & 0,0108 & 2,47 \\
\hline & 45 & 1,4279 & 0,1034 & 0,8893 & 0,9991 & 0,0022 & 1,09 \\
\hline & 55 & 1,0911 & 0,1003 & 0,8677 & 0,9969 & 0,0062 & 1,96 \\
\hline & 65 & 1,1354 & 0,0752 & 0,9025 & 0,9980 & 0,0030 & 1,90 \\
\hline \multirow{8}{*}{ Mizrahi \& Berk } & Temp. $\left({ }^{\circ} \mathbf{C}\right)$ & $\mathrm{K}_{\mathrm{oM}}(\mathrm{Pa})$ & $\mathrm{K}_{\mathrm{M}}(\text { Pa.s })^{\mathrm{n}}$ & $\mathbf{n}_{\mathrm{M}}$ & $\mathbf{R}^{2}$ & $\mathrm{X}^{2}$ & P (\%) \\
\hline & 5 & 1,4154 & 0,2093 & 0,5105 & 0,9992 & 0,0002 & 0,43 \\
\hline & 15 & 1,2457 & 0,2434 & 0,4651 & 0,9989 & 0,0002 & 0,55 \\
\hline & 25 & 1,1492 & 0,1918 & 0,4874 & 0,9949 & 0,0009 & 1,25 \\
\hline & 35 & 1,2440 & 0,0856 & 0,6275 & 0,9967 & 0,0005 & 0,99 \\
\hline & 45 & 1,1423 & 0,0773 & 0,6630 & 0,9989 & 0,0002 & 0,64 \\
\hline & 55 & 0,9673 & 0,0969 & 0,6092 & 0,9976 & 0,0004 & 0,91 \\
\hline & 65 & 0,9943 & 0,0760 & 0,6398 & 0,9988 & 0,0002 & 0,66 \\
\hline
\end{tabular}


Comportamento reológico das polpas de noni integral e concentradas

Sousa, S. F. et al.

Tabela 2. Parâmetros, coeficientes de determinação $\left(R^{2}\right)$, valores de Qui-quadrado $\left(X^{2}\right)$ e desvios percentuais médios (P\%) dos modelos reológicos ajustados aos reogramas da polpa concentrada de noni a $30^{\circ}$ Brix.

\begin{tabular}{|c|c|c|c|c|c|c|c|}
\hline \multirow{2}{*}{ Modelo } & \multirow{2}{*}{ Temp. $\left({ }^{\circ} \mathrm{C}\right)$} & \multicolumn{2}{|c|}{ Parâmetros } & \multirow{2}{*}{\multicolumn{2}{|c|}{$\mathbf{R}^{2}$}} & \multirow{2}{*}{$\mathrm{X}^{2}$} & \multirow{2}{*}{$\mathbf{P}(\%)$} \\
\hline & & $\mathrm{K}(\text { Pa.s })^{\mathrm{n}}$ & $\mathbf{n}$ & & & & \\
\hline \multirow{7}{*}{$\begin{array}{l}\text { Ostwald-de-Waelle } \\
\text { (Lei da Potência) }\end{array}$} & 5 & 109,9533 & 0,1743 & & 0,9942 & 6,0135 & 1,16 \\
\hline & 15 & 107,8461 & 0,1617 & & 0,9990 & 0,9367 & 0,46 \\
\hline & 25 & 101,9545 & 0,1619 & & 0,9984 & 1,2700 & 0,53 \\
\hline & 35 & 91,5396 & 0,1708 & & 0,9964 & 2,6420 & 0,86 \\
\hline & 45 & 88,3078 & 0,1679 & & 0,9984 & 1,1279 & 0,62 \\
\hline & 55 & 85,0295 & 0,1655 & & 0,9976 & 1,4718 & 0,78 \\
\hline & 65 & 76,5240 & 0,1678 & & 0,9950 & 2,6432 & 1,11 \\
\hline \multirow{8}{*}{ Herschel-Bulkley } & Temp. $\left({ }^{\circ} \mathbf{C}\right)$ & $\tau_{0 H}(\mathrm{~Pa})$ & $K_{H}(\text { Pa.s })^{n}$ & $\mathbf{n}_{\mathrm{H}}$ & $\mathbf{R}^{2}$ & $\mathrm{X}^{2}$ & P (\%) \\
\hline & 5 & 62,4025 & 52,0335 & 0,2828 & 0,9962 & 3,2285 & 1,00 \\
\hline & 15 & 27,1862 & 82,1965 & 0,1944 & 0,9985 & 1,2697 & 0,58 \\
\hline & 25 & 14,3503 & 88,4292 & 0,1782 & 0,9982 & 1,4034 & 0,56 \\
\hline & 35 & 45,4219 & 49,5925 & 0,2536 & 0,9978 & 1,5459 & 0,62 \\
\hline & 45 & 25,0980 & 64,8978 & 0,2061 & 0,9986 & 0,9159 & 0,50 \\
\hline & 55 & 16,2330 & 70,2307 & 0,1868 & 0,9973 & 1,6641 & 0,76 \\
\hline & 65 & 32,5401 & 47,3535 & 0,2237 & 0,9881 & 6,1717 & 1,72 \\
\hline \multirow{8}{*}{ Mizrahi \& Berk } & Temp. $\left({ }^{\circ} \mathbf{C}\right)$ & $\mathrm{K}_{\mathrm{oM}}(\mathrm{Pa})$ & $K_{M}(\text { Pa.s })^{n}$ & $\mathbf{n}_{\mathrm{m}}$ & $\mathbf{R}^{2}$ & $\mathrm{X}^{2}$ & $\mathbf{P}(\%)$ \\
\hline & 5 & 6,5947 & 4,0689 & 0,1797 & 0,9964 & 0,0047 & 0,49 \\
\hline & 15 & 6,6453 & 3,9252 & 0,1700 & 0,9967 & 0,0043 & 0,44 \\
\hline & 25 & 5,7602 & 4,4718 & 0,1526 & 0,9964 & 0,0037 & 0,37 \\
\hline & 35 & 5,8268 & 3,9132 & 0,1683 & 0,9996 & 0,0026 & 0,31 \\
\hline & 45 & 5,8820 & 3,7697 & 0,1650 & 0,9961 & 0,0037 & 0,39 \\
\hline & 55 & 5,8743 & 3,5361 & 0,1699 & 0,9811 & 0,0045 & 0,47 \\
\hline & 65 & 5,9571 & 3,0910 & 0,1787 & 0,9844 & 0,0171 & 0,95 \\
\hline
\end{tabular}

Tabela 3. Parâmetros, coeficientes de determinação $\left(R^{2}\right)$, valores de Qui-quadrado $\left(X^{2}\right)$ e desvios percentuais médios (P\%) dos modelos reológicos ajustados aos reogramas da polpa concentrada de noni a $50^{\circ}$ Brix.

\begin{tabular}{|c|c|c|c|c|c|c|c|}
\hline \multirow{2}{*}{ Modelo } & \multirow{2}{*}{ Temp. $\left({ }^{\circ} \mathrm{C}\right)$} & \multicolumn{2}{|c|}{ Parâmetros } & \multirow{2}{*}{\multicolumn{2}{|c|}{$\mathbf{R}^{2}$}} & \multirow{2}{*}{$\mathrm{X}^{2}$} & \multirow{2}{*}{$\mathbf{P}(\%)$} \\
\hline & & $K(\text { Pa.s })^{n}$ & $\mathbf{n}$ & & & & \\
\hline \multirow{7}{*}{$\begin{array}{l}\text { Ostwald-de-Waelle } \\
\text { (Lei da Potência) }\end{array}$} & 5 & 170,8070 & 0,2463 & & 0,9934 & 1,3169 & 1,58 \\
\hline & 15 & 157,5850 & 0,2442 & & 0,9904 & 2,3239 & 1,95 \\
\hline & 25 & 143,1720 & 0,2477 & & 0,9986 & 6,7021 & 2,03 \\
\hline & 35 & 126,6320 & 0,2803 & & 0,9904 & 4,4648 & 2,17 \\
\hline & 45 & 120,2170 & 0,2944 & & 0,9896 & 2,1718 & 2,41 \\
\hline & 55 & 99,2276 & 0,3236 & & 0,9920 & 2,8782 & 2,62 \\
\hline & 65 & 93,1389 & 0,3292 & & 0,9932 & 2,5577 & 2,44 \\
\hline \multirow{8}{*}{ Herschel-Bulkley } & Temp. $\left({ }^{\circ} \mathbf{C}\right)$ & $\tau_{0 H}(\mathbf{P a})$ & $K_{H}(\text { Pa.s })^{n}$ & $n_{H}$ & $\mathbf{R}^{2}$ & $\mathbf{X}^{2}$ & $\mathbf{P}(\%)$ \\
\hline & 5 & 135,2241 & 42,8726 & 0,5294 & 0,9559 & 1,3612 & 4,54 \\
\hline & 15 & 186,4556 & 12,2774 & 0,7740 & 0,9050 & 2,7612 & 7,51 \\
\hline & 25 & 148,5862 & 21,4693 & 0,6251 & 0,9634 & 4,7081 & 4,73 \\
\hline & 35 & 145,9619 & 15,8208 & 0,7175 & 0,9444 & 2,2845 & 6,96 \\
\hline & 45 & 139,8779 & 17,0610 & 0,7039 & 0,9354 & 3,1595 & 8,25 \\
\hline & 55 & 122,0091 & 10,6340 & 0,8253 & 0,9240 & 3,5332 & 10,18 \\
\hline & 65 & 93,3784 & 21,7464 & 0,6344 & 0,9630 & 2,7776 & 6,71 \\
\hline \multirow{8}{*}{ Mizrahi \& Berk } & Temp. $\left({ }^{\circ} \mathrm{C}\right)$ & $\mathrm{K}_{\mathrm{om}}(\mathrm{Pa})$ & $K_{M}(\text { Pa.s })^{n}$ & $\mathbf{n}_{\mathrm{M}}$ & $\mathbf{R}^{2}$ & $\mathbf{X}^{2}$ & P (\%) \\
\hline & 5 & 7,7514 & 5,8340 & 0,2122 & 0,8219 & 0,1026 & 1,63 \\
\hline & 15 & 6,2721 & 6,6757 & 0,1839 & 0,9725 & 0,1432 & 1,87 \\
\hline & 25 & 8,4820 & 4,1541 & 0,2484 & 0,9852 & 0,0709 & 1,19 \\
\hline & 35 & 7,1918 & 4,4943 & 0,2560 & 0,9821 & 0,1017 & 1,84 \\
\hline & 45 & 7,1953 & 4,2039 & 0,2813 & 0,9814 & 0,1161 & 1,96 \\
\hline & 55 & 7,3469 & 3,0324 & 0,3535 & 0,9763 & 0,1569 & 2,26 \\
\hline & 65 & 6,0305 & 3,8726 & 0,2977 & 0,9790 & 0,1390 & 2,18 \\
\hline
\end{tabular}


Viotto (2015) também verificaram os melhores ajustes com esse modelo, ao estudarem o efeito do tratamento enzimático no suco de pitanga, encontraram valores de $\chi^{2}$ iguais a zero em todas as amostras.

A tensão de cisalhamento inicial $\left(\tau_{\mathrm{OH}}\right.$ e $\left.\mathrm{K}_{\mathrm{OM}}\right)$, que, segundo Steffe (1996), é uma tensão finita necessária para que o fluido comece a escoar, geralmente diminui com o aumento da temperatura; no entanto, este decréscimo não é uma regra para os modelos reológicos, não sendo também observado no trabalho de Pereira et al. (2012), ao analisarem o comportamento reológico da polpa de goiaba cv. Paluma nas temperaturas de 10 a $50{ }^{\circ} \mathrm{C}$, com ajustes pelos modelos de Herschel-Bulkley e Mizrahi \& Berk.

Em relação ao índice de consistência $(K)$, este indica o grau de resistência do fluido ao escoamento. Observa-se que $\mathrm{K}$ apresentou tendência de redução com o aumento da temperatura para as polpas integral e concentradas de noni, indicando que estas se tornaram menos consistentes. Este comportamento também foi observado por Sousa et al. (2014), ao avaliarem o efeito da temperatura sobre o comportamento reológico da polpa de pequi (Caryocar coriaceum) com diferentes concentrações de sólidos.

O índice de comportamento do fluido (n) apresenta-se com valores inferiores à unidade $(n<1)$, caracterizando, portanto, os fluidos como não newtonianos e pseudoplásticos. A pseudoplasticidade é um comportamento característico para a maioria das polpas de frutas, sendo também observado por Oliveira et al. (2011), para as polpas de gabiroba e goiaba, nas temperaturas de 20 a $35^{\circ} \mathrm{C}$; por Oliveira et al. (2012), para a polpa de morango (6,80 $\mathrm{Brix})$, nas temperaturas de 20 a $35^{\circ} \mathrm{C}$; e por Quek et al. (2013), para a polpa de graviola em diferentes concentrações (10-50 Brix) e temperaturas de 10 a $60^{\circ} \mathrm{C}$ ).

A Figura 1 mostra os reogramas das polpas integral e concentradas nas temperaturas de 5 a $65^{\circ} \mathrm{C}$, com as curvas ajustadas com o modelo de Mizrahi \& Berk, considerado como o melhor modelo dentre os avaliados.

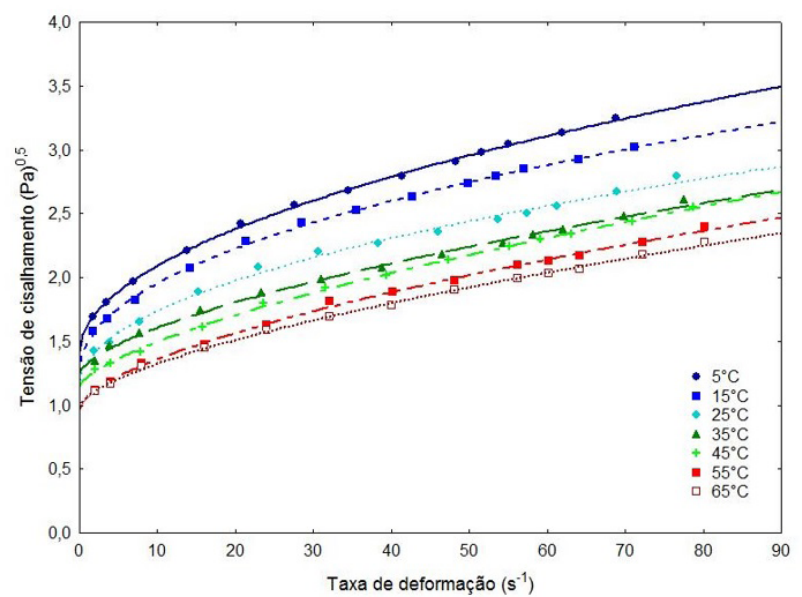

(a)

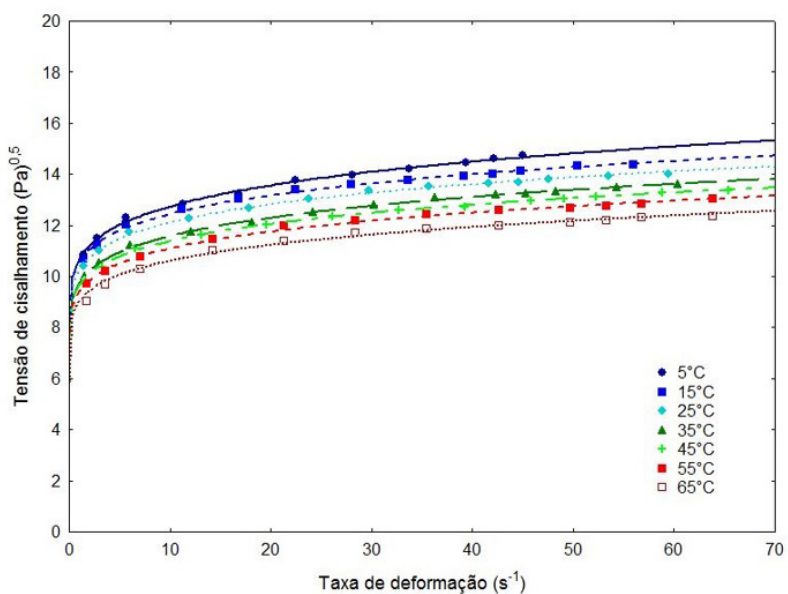

(b)

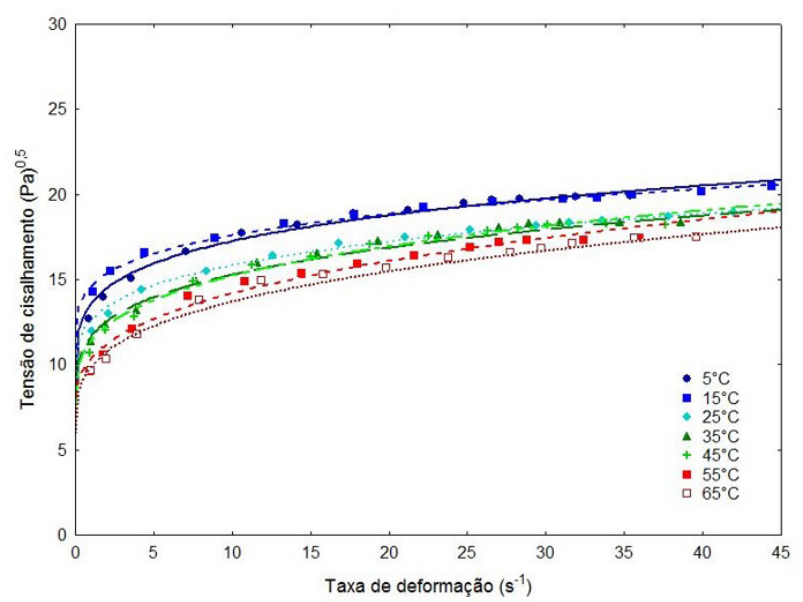

(c)

Figura 1. Reogramas das polpas de noni em diferentes temperaturas com o modelo de Mizrahi \& Berk ajustado aos dados experimentais: (a) polpa integral; (b) polpa a $30^{\circ}$ Brix; (c) polpa a $50^{\circ} \mathrm{Brix}$. 
De maneira geral, os menores valores para a tensão de cisalhamento são observados nas temperaturas mais altas a uma dada taxa de deformação fixa, indicando que a viscosidade aparente diminui com o aumento da temperatura. Esse comportamento é semelhante aos resultados obtidos por Oliveira et al. (2012), para a polpa de morango, em que ocorreram variações da viscosidade aparente com a variação da tensão de cisalhamento nas diversas temperaturas estudadas, percebendo-se que a viscosidade aparente foi maior nas temperaturas mais baixas. Observa-se que as polpas de noni, por ser um fluido pseudoplástico, não apresentam uma relação linear entre a tensão de cisalhamento e a taxa de deformação.

Nas Tabelas 4-6, são mostradas as médias para a viscosidade aparente das polpas integral e concentradas em função da velocidade de rotação e da temperatura. Observou-se que, com o aumento da velocidade de rotação (5 a 200 rpm) e da temperatura $\left(5\right.$ a $\left.65^{\circ} \mathrm{C}\right)$, reduziram-se os valores médios de viscosidade aparente, comportamento típico dos fluidos pseudoplásticos. Lima et al. (2014), igualmente, verificaram que a viscosidade aparente da polpa de abacaxi diminuiu com o aumento da velocidade de rotação (1,5 a 60 rpm), nas temperaturas de 30 e $40^{\circ} \mathrm{C}$.

Este comportamento pode ser justificado pela mudança estrutural da amostra decorrente das forças geradas, o que faz com que as partículas se rearranjem em direções paralelas, ocorrendo a quebra de partículas grandes em partículas menores, sendo que estas podem escoar mais facilmente na direção da tensão aplicada (KESHANI et al., 2012; SHAMSUDIN et al., 2013). Deshmukh et al. (2015) relataram que o aumento da temperatura dos fluidos leva ao aumento da mobilidade das moléculas e aumenta os espaços intermoleculares, o que promove o decréscimo da resistência ao fluxo, com consequente redução da viscosidade.

Tabela 4. Viscosidade aparente (Pa.s) para a polpa integral de noni.

\begin{tabular}{cccccccc} 
Velocidade de & \multicolumn{7}{c|}{ Temperatura $\left.\mathbf{~}^{\circ} \mathbf{C}\right)$} \\
\cline { 2 - 8 } rotação $(\mathbf{r p m})$ & $\mathbf{5}$ & $\mathbf{1 5}$ & $\mathbf{2 5}$ & $\mathbf{3 5}$ & $\mathbf{4 5}$ & $\mathbf{5 5}$ & $\mathbf{6 5}$ \\
$\mathbf{5}$ & 1,666 & 1,406 & 1,063 & 0,928 & 0,832 & 0,623 & 0,611 \\
$\mathbf{1 0}$ & 0,946 & 0,793 & 0,582 & 0,551 & 0,449 & 0,347 & 0,338 \\
$\mathbf{2 0}$ & 0,564 & 0,465 & 0,355 & 0,315 & 0,255 & 0,221 & 0,212 \\
$\mathbf{4 0}$ & 0,355 & 0,302 & 0,233 & 0,196 & 0,165 & 0,135 & 0,131 \\
$\mathbf{6 0}$ & 0,284 & 0,243 & 0,189 & 0,152 & 0,138 & 0,110 & 0,104 \\
$\mathbf{8 0}$ & 0,240 & 0,207 & 0,158 & 0,127 & 0,117 & 0,103 & 0,090 \\
$\mathbf{1 0 0}$ & 0,209 & 0,180 & 0,134 & 0,111 & 0,104 & 0,089 & 0,079 \\
$\mathbf{1 2 0}$ & 0,188 & 0,162 & 0,121 & 0,102 & 0,097 & 0,081 & 0,075 \\
$\mathbf{1 4 0}$ & 0,176 & 0,151 & 0,112 & 0,095 & 0,091 & 0,078 & 0,070 \\
$\mathbf{1 5 0}$ & 0,172 & 0,146 & 0,109 & 0,093 & 0,089 & 0,075 & 0,069 \\
$\mathbf{1 6 0}$ & 0,168 & 0,142 & 0,107 & 0,090 & 0,087 & 0,073 & 0,066 \\
$\mathbf{1 8 0}$ & 0,158 & 0,133 & 0,103 & 0,088 & 0,084 & 0,072 & 0,066 \\
$\mathbf{2 0 0}$ & 0,153 & 0,128 & 0,102 & 0,087 & 0,082 & 0,071 & 0,064 \\
\hline
\end{tabular}

Tabela 5. Viscosidade aparente (Pa.s) para a polpa de noni concentrada a $30^{\circ}$ Brix.

\begin{tabular}{|c|c|c|c|c|c|c|c|}
\hline \multirow{2}{*}{$\begin{array}{l}\text { Velocidade de } \\
\text { rotação (rpm) }\end{array}$} & \multicolumn{7}{|c|}{ Temperatura $\left({ }^{\circ} \mathbf{C}\right)$} \\
\hline & 5 & 15 & 25 & 35 & 45 & 55 & 65 \\
\hline 5 & 83,909 & 81,355 & 72,900 & 66,350 & 59,073 & 53,267 & 46,117 \\
\hline 10 & 47,223 & 45,042 & 40,960 & 36,703 & 32,795 & 29,237 & 26,479 \\
\hline 20 & 26,915 & 25,780 & 23,144 & 20,828 & 18,829 & 16,384 & 14,883 \\
\hline 40 & 14,663 & 14,233 & 12,686 & 11,393 & 10,295 & 9,246 & 8,539 \\
\hline 60 & 10,398 & 10,099 & 9,038 & 8,071 & 7,358 & 6,730 & 6,097 \\
\hline 80 & 8,412 & 8,001 & 7,154 & 6,478 & 5,815 & 5,235 & 4,816 \\
\hline 100 & 6,930 & 6,591 & 6,024 & 5,434 & 4,837 & 4,358 & 3,965 \\
\hline 120 & 5,973 & 5,644 & 5,139 & 4,721 & 4,126 & 3,713 & 3,380 \\
\hline 140 & 5,299 & 4,954 & 4,486 & 4,119 & 3,675 & 3,233 & 2,947 \\
\hline 150 & 5,074 & 4,679 & 4,216 & 3,872 & 3,464 & 3,052 & 2,794 \\
\hline 160 & 4,828 & 4,464 & 4,006 & 3,677 & 3,292 & 2,906 & 2,663 \\
\hline 180 & - & 4,069 & 3,638 & 3,342 & 3,001 & 2,666 & 2,384 \\
\hline 200 & - & 3,687 & 3,302 & 3,068 & 2,724 & 2,431 & 2,164 \\
\hline
\end{tabular}


Comportamento reológico das polpas de noni integral e concentradas

Sousa, S. F. et al.

Com relação à concentração, as polpas de noni apresentaram um aumento expressivo nos valores de viscosidade aparente, tendo, estes mesmos valores, sido influenciados pelos demais parâmetros (temperatura e velocidade de rotação).

Na Figura 2, apresentam-se os gráficos do comportamento da viscosidade aparente em função da velocidade de rotação e da temperatura, para as polpas integral e concentradas.

Miranda et al. (2012), ao estudarem a polpa da graviola em diferentes temperaturas (5, 10, 20, 30, 40, $50{ }^{\circ} \mathrm{C}$ ), velocidades de rotação (50 e $200 \mathrm{rpm}$ ) e concentrações (12, 17 e 25ํix), observaram que, em todas as concentrações, as viscosidades aparentes das

Tabela 6. Viscosidade aparente (Pa.s) para a polpa de noni concentrada a $50^{\circ}$ Brix.

\begin{tabular}{cccrrrrr} 
Velocidade de & \multicolumn{7}{c}{ Temperatura $\left({ }^{\circ} \mathbf{C}\right)$} \\
\cline { 2 - 8 } rotação $(\mathbf{r p m})$ & $\mathbf{5}$ & $\mathbf{1 5}$ & $\mathbf{2 5}$ & $\mathbf{3 5}$ & $\mathbf{4 5}$ & $\mathbf{5 5}$ & $\mathbf{6 5}$ \\
\hline $\mathbf{5}$ & 182,554 & 150,48 & 136,267 & 133,762 & 122,128 & 102,356 & 94,465 \\
$\mathbf{1 0}$ & 109,785 & 94,338 & 80,533 & 78,632 & 77,298 & 61,911 & 54,162 \\
$\mathbf{2 0}$ & 64,068 & 55,856 & 49,200 & 44,974 & 43,638 & 40,678 & 35,212 \\
$\mathbf{4 0}$ & 38,994 & 32,810 & 28,600 & 28,653 & 29,340 & 27,417 & 24,076 \\
$\mathbf{6 0}$ & 29,608 & 25,942 & 21,378 & 21,979 & 22,340 & 20,430 & 18,785 \\
$\mathbf{8 0}$ & 23,393 & 20,426 & 17,517 & 17,663 & 17,816 & 16,392 & 14,813 \\
$\mathbf{1 0 0}$ & 20,201 & 16,800 & 14,520 & 15,393 & 15,519 & 14,016 & 12,444 \\
$\mathbf{1 0}$ & 17,072 & 14,586 & 12,767 & 13,371 & 13,665 & 12,444 & 11,136 \\
$\mathbf{1 4 0}$ & 15,367 & 13,046 & 11,190 & 12,031 & 12,149 & 11,311 & 9,939 \\
$\mathbf{1 5 0}$ & 14,554 & 12,310 & 10,658 & 11,529 & 11,548 & 10,941 & 9,531 \\
$\mathbf{1 0 0}$ & 13,763 & 11,703 & 10,142 & 10,863 & 11,003 & 10,393 & 9,271 \\
$\mathbf{1 8 0}$ & 12,418 & 10,514 & 9,274 & 9,696 & 9,855 & 9,273 & 8,508 \\
$\mathbf{2 0 0}$ & 11,216 & 9,606 & 8,553 & 8,705 & 8,832 & 8,423 \\
\hline
\end{tabular}

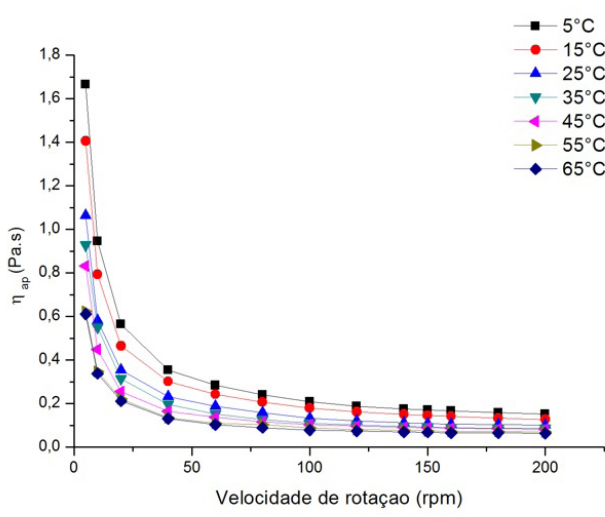

(a)

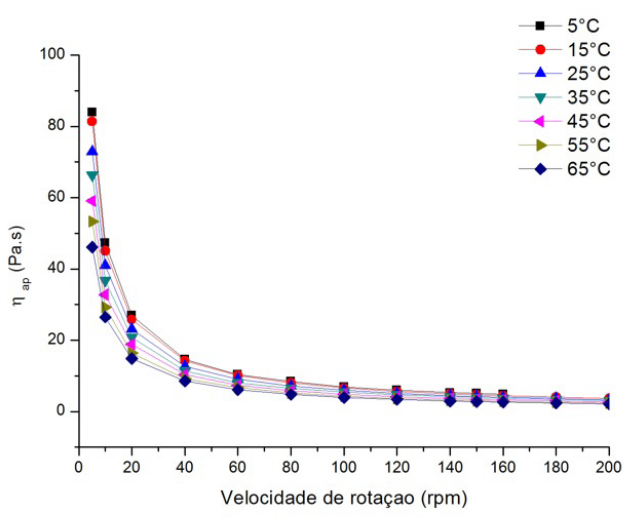

(b)

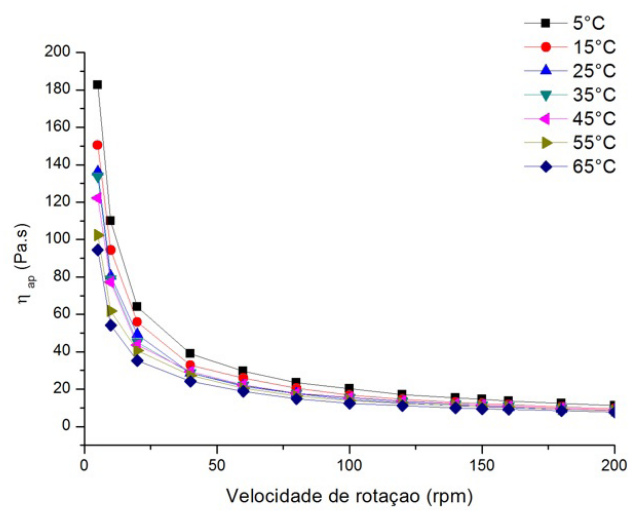

(c)

Figura 2. Viscosidade aparente das polpas de noni em função da velocidade de rotação: (a) polpa integral de noni; (b) polpa com $30^{\circ}$ Brix; (c) polpa com $50^{\circ}$ Brix. 
polpas foram influenciadas pelas velocidades de rotação e temperaturas. Verificaram que as viscosidades diminuíram com o aumento dessas variáveis, como também houve aumento das viscosidades aparentes com o aumento do teor de sólidos solúveis totais ( ${ }^{\circ}$ Brix) das amostras.

Na Figura 3, mostra-se a aplicação da equação do tipo Arrhenius linearizada na forma de In da viscosidade aparente em função do inverso da temperatura $(\mathrm{K})$ para as polpas do noni integral e concentradas. Observa-se uma tendência de redução da viscosidade aparente com o aumento da temperatura e da velocidade de rotação.

De acordo com Steffe (1996), a energia de ativação indica a influência da temperatura na viscosidade aparente dos fluidos. A Tabela 7 mostra os valores da energia

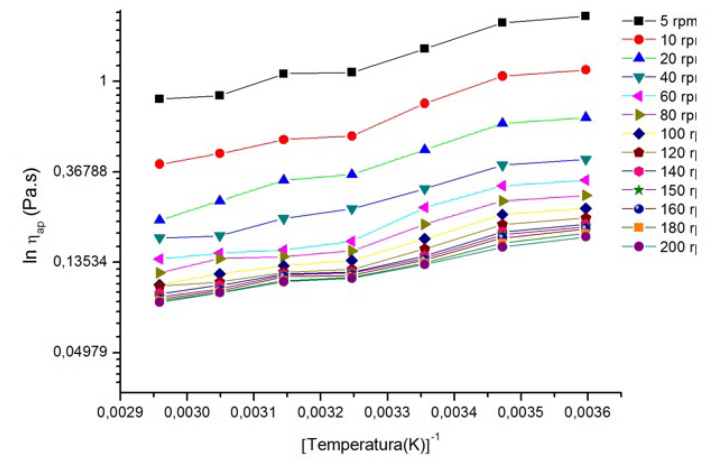

(a)

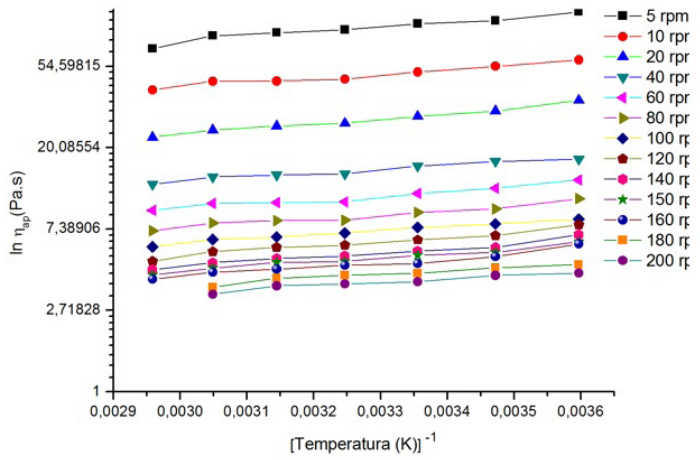

(b)

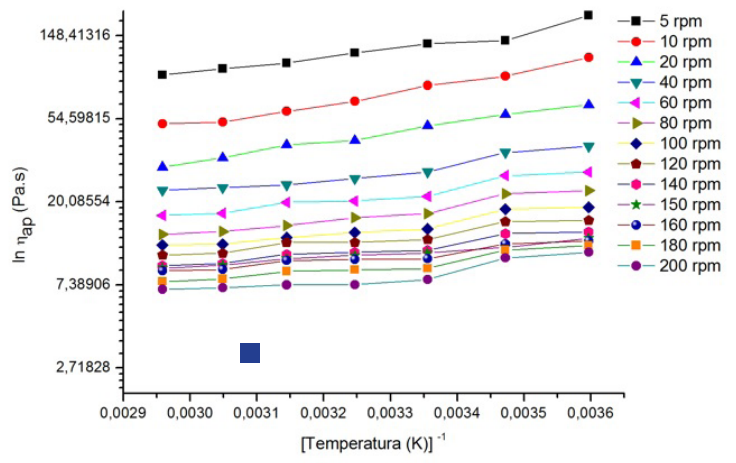

(c)

Figura 3. Viscosidade aparente das polpas de noni em função do inverso da temperatura: (a) polpa integral de noni; (b) polpa com $30^{\circ}$ Brix; (c) polpa com $50^{\circ}$ Brix.

Tabela 7. Energia de ativação e coeficientes de determinação $\left(R^{2}\right)$ para as polpas integral e concentradas (30 e $50^{\circ}$ Brix) de noni.

\begin{tabular}{|c|c|c|c|c|c|c|}
\hline \multirow{2}{*}{$\begin{array}{l}\text { Velocidade de } \\
\text { rotação (rpm) }\end{array}$} & \multicolumn{2}{|c|}{ Polpa integral } & \multicolumn{2}{|c|}{ Polpa com $30^{\circ}$ Brix } & \multicolumn{2}{|c|}{ Polpa com $50^{\circ}$ Brix } \\
\hline & $E_{a}\left(k^{\prime} g^{-1} \mathrm{~mol}^{-1}\right)$ & $\mathbf{R}^{2}$ & $\mathrm{E}_{\mathrm{a}}\left(\mathrm{kJ} \mathrm{g}^{-1} \mathrm{~mol}^{-1}\right)$ & $\mathbf{R}^{2}$ & $E_{a}\left(k^{J} g^{-1} \mathrm{~mol}^{-1}\right)$ & $\mathbf{R}^{2}$ \\
\hline 5 & 14,0126 & 0,9067 & 509,9994 & 0,9657 & 1051,4820 & 0,8961 \\
\hline 10 & 8,0430 & 0,8993 & 284,2861 & 0,9799 & 658,1432 & 0,8774 \\
\hline 20 & 4,6474 & 0,8857 & 164,9508 & 0,9775 & 345,0023 & 0,9151 \\
\hline 40 & 3,0273 & 0,9134 & 85,7802 & 0,9704 & 155,2911 & 0,6162 \\
\hline 60 & 2,4248 & 0,9134 & 59,4952 & 0,9708 & 120,7846 & 0,6284 \\
\hline 80 & 1,9797 & 0,8892 & 49,3286 & 0,9859 & 94,7817 & 0,7058 \\
\hline 100 & 1,6991 & 0,8731 & 40,5524 & 0,9817 & 78,7712 & 0,5477 \\
\hline 120 & 1,4859 & 0,8588 & 35,2725 & 0,9819 & 59,8505 & 0,5352 \\
\hline 140 & 1,3632 & 0,8455 & 31,5378 & 0,9902 & 53,2449 & 0,4640 \\
\hline 150 & 1,3317 & 0,8519 & 30,3037 & 0,9960 & 47,9017 & 0,4246 \\
\hline 160 & 1,3137 & 0,8534 & 28,8513 & 0,9958 & 43,2658 & 0,4123 \\
\hline 180 & 1,1898 & 0,8669 & 27,0413 & 0,9970 & 38,8188 & 0,4545 \\
\hline 200 & 1,1309 & 0,8821 & 24,4689 & 0,9938 & 35,9055 & 0,5360 \\
\hline
\end{tabular}


de ativação $\left(\mathrm{kJ} \mathrm{g}^{-1} \mathrm{~mol}^{-1}\right)$ das polpas de noni e seus respectivos coeficientes de determinação $\left(R^{2}\right)$. A polpa de noni integral apresentou $\mathrm{R}^{2}$ entre 0,8455 e 0,9134; a polpa concentrada a $30^{\circ}$ Brix apresentou $R^{2}$ com valores entre 0,9704 e 0,9970, e na polpa com $50^{\circ}$ Brix, o ajuste ficou entre 0,4123 e 0,9151. Esses valores indicam que a equação tipo Arrhenius pode ser utilizada para demonstrar o efeito da temperatura sobre a viscosidade aparente nas polpas integral e com $30^{\circ} \mathrm{Brix}$, em todas as velocidades de rotação, e na polpa com $50^{\circ}$ Brix, preferencialmente nas velocidades de rotação de 5, 10 e $20 \mathrm{rpm}\left(R^{2}>0,80\right)$.

Feitosa et al. (2015), trabalhando com a polpa de murta integral, obtiveram coeficiente de determinação $\left(R^{2}\right)$ superiores a 0,78, ao ajustarem os dados à equação de Arrhenius. Chin et al. (2009) relataram valores de coeficiente de determinação superiores a 0,94 para a equação de Arrhenius aplicada aos dados para a polpa de pomelo, em função das concentrações de sólidos solúveis totais 20, 30 e $50^{\circ}$ Brix.

As polpas de noni apresentaram os valores mais elevados para a energia de ativação a velocidades baixas, indicando que a temperatura exerce maior efeito sobre a viscosidade aparente nas baixas taxas de deformação. Logo, o aumento da velocidade de rotação promoveu uma diminuição na energia de ativação (Ea). Resultados parecidos foram observados por Feitosa et al. (2015), ao estudarem a polpa da murta integral, quando verificaram que a energia de ativação (Ea) reduziu de 24,4983 para $8,4759 \mathrm{~kJ} \mathrm{~mol}^{-1}$, em um intervalo da taxa de deformação entre 5,6 a $28 \mathrm{~s}^{-1}$.

Para uma velocidade de rotação fixa, observa-se que a $\mathrm{E}_{\mathrm{a}}$ aumenta com o aumento do teor de sólidos solúveis totais, indicando um maior efeito da temperatura nas polpas mais concentradas, de acordo com Deshmukh et al. (2015), os quais verificaram que, quanto maior o teor de sólidos solúveis totais do suco de sapoti clarificado, maior a $\mathrm{E}_{\mathrm{a}}$ $\left(5,218\right.$ a $25,439 \mathrm{~kJ} \mathrm{~mol}^{-1}$ para 10,2 a 55,6³rix).

\section{Conclusões}

A caracterização do comportamento reológico demonstrou haver diferenças entre as polpas de noni integral e concentradas. O modelo reológico de Mizrahi \& Berk foi o melhor dentre os avaliados para descrever o comportamento das amostras. As polpas foram classificadas como fluidos não newtonianos pseudoplásticos $(n<1)$. O aumento da velocidade de rotação e da temperatura acarretaram a diminuição da viscosidade aparente das polpas, o que facilita o escoamento e a troca de calor durante processos térmicos. A polpa integral apresentou menores valores para a viscosidade aparente, em todas as condições estudadas, necessitando de menor tensão para escoar, como pode ser comprovado pelos modelos testados (Herschel-Bulkley e Mizrahi \& Berk). Tal fato deve ser considerado em eventuais dimensionamentos de tubulações e bombas. A equação tipo Arrhenius apresentou um bom ajuste para demonstrar o efeito da temperatura sobre a viscosidade aparente nas polpas integral e concentrada de noni a $30^{\circ}$ Brix.

\section{Referências}

BRAGA, A. C. C.; RODRIGueS, A. M. C.; SILVA, L. H. M.; ARAÚJO, L. A. Avaliação da influência da temperatura e do tratamento enzimático no comportamento reológico do suco de abacaxi pérola (Ananas comosus L. Merr.). Revista Brasileira de Fruticultura, São Paulo, v. 35, n. 1, p. 226-237, 2013.

BRANCO, I. G. Suco de laranja concentrado: comportamento reológico a baixas temperaturas. 1995. 91 f. Dissertação (Mestrado em Engenharia de Alimentos)-Faculdade de Engenharia de Alimentos, Universidade de Campinas, Campinas, 1995.

CHIN, N. L.; CHAN, S. M.; YUSOF, Y. A.; CHUAH, T. G.; TALIB, R. A. Modelling of rheological behaviour of pummelo juice concentrates using mastercurve. Journal of Food Engineering, London, v. 93, n. 2, p. 134-140, 2009. http://dx.doi.org/10.1016/j. jfoodeng.2009.01.005.

DESHMUKH, P. S.; MANJUNATHA, S. S.; RAJU, P. S. Rheological behaviour of enzyme clarified sapota (Achras sapota $L$ ) juice at different concentration and temperatures. Journal of Food Science and Technology, Oxford, v. 52, n. 4, p. 1896-1910, 2015. PMid:25829571. http://dx.doi.org/10.1007/s13197-013-1222-5.

FEITOSA, R. M.; FIGUEIRÊDO, R. M. F.; QUEIROZ, A. J. M.; SOUZA, E. P.; SILVA, V. M. Viscosidade aparente da polpa de murta integral em diferentes temperaturas. Revista Caatinga, Mossoró, v. 28, n. 4, p. 235-243, 2015.

KESHANI, S.; LUQMAN, A. C.; RUSSLY, A. R. Effect of temperature and concentration on rheological properties pomelo juice concentrates. International Food Research Journal, Selangor, v. 19, n. 2, p. 553-562, 2012.

LIMA, F. C. S.; MELO, J. C. S.; PEREIRA, E. D.; COSTA, C. H. C.; MIRANDA, V. A. M. Estudo da viscosidade aparente da polpa de abacaxi integral. In: CONGRESSO BRASILEIRO DE QUÍMICA, 54., 2014, Natal. Anais eletrônicos... Natal: ABQ, 2014. Disponível em: <www.abq.org.br/cbq/2014/trabalhos/10/5766-17673.html>. Acesso em: 10 jun. 2016.

MIRANDA, V. A. M.; QUEIROZ, A. J. M.; FIGUEIRÊDO, R. M. F.; SANTOS, D. C. Viscosidade aparente de polpas de graviola com diferentes concentrações. Revista Brasileira de Produtos Agroindustriais, Campina Grande, v. 14, p. 423-434, 2012.

MITSCHKA, P. Simple conversion of Brookfield RVT: readings into viscosity functions. Rheologica Acta, Prague, v. 21, p. 207-209, 1982.

NASCIMENTO, L. C. S. Caracterização centesimal, composição química e atividade cantioxidante do noni (Morinda citrifolia L.) cultivado no Município de Zé Doca-MA. 2012. 69 f. Dissertação (Mestrado em Ciência e Tecnologia de Alimentos). Universidade Federal Rural do Rio de Janeiro, Seropédica, 2012. 
OLIVEIRA, R. C.; ROSSI, R. M.; BARROS, S. T. D. Estudo do efeito da temperatura sobre o comportamento reológico das polpas de gabiroba e goiaba. Acta Scientiarum. Technology, Maringá, v. 33, n. 1, p. 31-37, 2011. http://dx.doi.org/10.4025/ actascitechnol.v33i1.5852.

OLIVEIRA, R. C.; ROSSI, R. M.; BARROS, S. T. D. Estudo reológico da polpa de morango (fragaria vesca) em diferentes temperaturas. Acta Scientiarum. Technology, Maringá, v. 34, n. 3, p. 283-288, 2012. http://dx.doi.org/10.4025/actascitechnol.v34i3.7833.

ONGARATTO, R.; VIOTTO, L. A. Efeito do tratamento enzimático sobre a viscosidade e os teores de fibra e pectina em suco de pitanga (Eugenia uniflora L.). Brazilian Journal of Food Technology, Campinas., v. 18, n. 3, p. 231-238, 2015. http:// dx.doi.org/10.1590/1981-6723.5514.

PEREIRA, J. L.; QUEIROZ, A. J. M.; FIGUEIRÊDO, R. M. F.; NUNES, J. T.; GOMES, J. P. Comportamento reológico de polpa de goiaba Cv. Paluma. Revista Brasileira de Produtos Agroindustriais, Campina Grande, v. 14, n. esp., p. 479-496, 2012.

PINO, J. A.; MARQUEZ, E.; CASTRO, D. Volatile and non-volatile acids of noni (Morinda citrifolia L.) fruit. Journal Science Food Agriculture, Cambridge, v. 88, n. 7, p. 1247-1249, 2009.
QUEK, M. C.; CHIN, N. L.; YUSOF, Y. A. Modelling of rheological behaviour of soursop juice concentrates using shear ratetemperature-concentration superposition. Journal of Food Engineering, Oxford, v. 118, n. 4, p. 380-386, 2013. http://dx.doi. org/10.1016/j.jfoodeng.2013.04.025.

SHAMSUDIN, R.; LING, C. S.; ADZAHAN, N. M.; DAUD, W. R. W. Rheological properties of ultraviolet-irradiated and thermally pasteurized Yankee pineapple juice. Journal of Food Engineering, Oxford, v. 116, n. 2, p. 548-553, 2013. http://dx.doi.org/10.1016/j. jfoodeng.2012.12.031.

SOUSA, E. P.; QUEIROZ, A. J. M.; FIGUEIRÊDO, R. M. F.; LEMOS, D. M. Comportamento reológico e efeito da temperatura da polpa de pequi em diferentes concentrações. Brazilian Journal of Food Techology, Campinas, v. 17, n. 3, p. 226-235, 2014. http://dx.doi.org/10.1590/1981-6723.1214.

STEFFE, J. F. Rheological methods in food process engineering. Michigan: Freeman Press, 1996. 428 p.

ZHONG, Q.; DAUBERT, C. R. Food rheology. In: KUTZ, M. (Ed.). Handbook of farm, dairy and food machinery. Heidelberg: Elsevier, 2007. p. 391-414. 